\title{
El currículo praxeológico como interés de conocimiento emancipatorio ${ }^{1}$
}

\author{
Edgar Pineda Martinez ${ }^{2}$ \\ Universidad Santo Tomás, Villavicencio, Colombia ${ }^{3}$ \\ edgarpin535@gmail.com \\ Paula Andrea Orozco Pineda ${ }^{4}$ \\ Corporación Universitaria Minuto de Dios —unImINUTO, Villavicencio, Colombia ${ }^{5}$ \\ paula.orozco@uniminuto.edu
}

1 Artículo de investigación producto del proyecto "Programa para el mejoramiento de las competencias comunicativas en estudiantes de primer año universitario", financiado por convocatoria interna de la Vicerrectoría General académica y la Dirección General de investigaciones de uniminuto.

2 Licenciado en Filosofía, Magister en Educación y Magister en Neuropsicología y educación,

3 Docente investigador miembro del grupo de Investigación A.B.A.

4 Licenciada en Lenguas Modernas, Magister en Educación con acentuación en procesos cognitivos.

5 Coordinadora de Unidad de Transversales de la Corporación Universitaria Minuto de Dios -UNIMINUTO, Vicerrectoría Regional Llanos, miembro del Grupo de Investigación Trabajo de Llano. 


\title{
El currículo praxeológico como interés de conocimiento emancipatorio
}

\section{Resumen}

Con miras a presentar alternativas de comprensión frente a la discusión sobre el currículo praxeológico, este artículo busca abrir horizontes de sentido sobre la relación entre currículo, praxeología, práctica y saber pedagógico en la educación superior. En un primer momento, se hace una aproximación a los principales escenarios que determinan de algún modo al currículo praxeológico desde la interdisciplina y la subjetividad en el ejercicio de la educación superior. Luego, en un segundo apartado, se presenta el currículo praxeológico como una posibilidad de transformación y de resignificación de la práctica pedagógica desde los conceptos de intereses de conocimiento de Habermas y de tipo de currículo de Grundy. Tercero, se presenta una comparación entre los conceptos de Habermas y Grundy sobre currículo e interés de conocimiento, el currículo praxeológico y las consideraciones, saberes e imaginarios de docentes universitarios. Finalmente, se presenta la necesidad de formación desde lo humano -en términos de la educación social, la educación popular y la praxeología- como opción fundamental del currículo praxeológico.

Palabras clave: currículo, educación superior, sujeto, subjetividad, praxeología.

\section{The praxeological curriculum as emancipatory interest of knowledge}

\begin{abstract}
With a view to present some understanding alternatives in front of the praxeological curriculum discussion, this article looks forward in the opening of horizons of sense about the relationship between curriculum, praxeology, praxis and pedagogical learning over the higher education. In a first step, it has to be done an approximation to the main sceneries that determine in any form the possibilities to the praxeological curriculum from the interdiscipline and the subjectivity in the higher education exercise. Then, in a second step, it is presented the praxeological curriculum with a possibility of transformation and a pedagogical practice re-significance since the knowledge interest's concepts of Habermas and from the Grundy'scurriculum type. Forward, it is presented a comparison between the concepts of Habermas and Grundy about the curriculum and the knowledge interest, the praxeological curriculum and its thoughtfulnesses, knowledges and universitarian teacher's notions. Finally, it is presented the need of formation since the human being, in terms of the social education, the popular education and the praxeology, as a fundamental option of the praxeological curriculum.
\end{abstract}

Keywords: curriculum; higher education; subject; subjectivity; praxeology.

\section{O currículo praxiológico como o interesse emancipatório do conhecimento}

\section{Resumo}

Tendo em vista apresentar alternativas de entendimento em frente à discussão sobre o currículo praxeológico, este artigo procura abrir horizontes de sentido sobre a relação entre currículo, praxeologia, prática e saber pedagógico na educação superior. Num primeiro momento, faz-se uma aproximação aos principais ambientes que determinam de algum modo ao currículo praxeológico desde a interdisciplina e a subjetividade no exercício da educação superior. Depois, num segundo apartado, apresenta-se o currículo praxeológico como uma possibilidade de transformação e de re significação da prática pedagógica desde os conceitos de interesses de conhecimento de Habermas e de tipo de currículo de Grundy. Terceiro, apresenta-se uma comparação entre os conceitos de Habermas e Grundy sobre currículo e interesse de conhecimento, o currículo praxeológico e as considerações, saberes e imaginários de docentes universitários. Finalmente, apresenta-se a necessidade de formação desde o humano -em termos da educação social, a educação popular e a praxeología - como opção fundamental do currículo praxeológico.

Palavras-chave: currículo, ensino superior, sujeito, subjetividade, Praxeologia 


\section{Introducción}

La práctica supone para Aristóteles praxis-quehacer-, la cual, superando la concepción de trabajo mecánico propio de la tecné, se introduce en un registro más amplio, esto es, la Phronesis, acción que implica interactividad, experiencia, compromiso y valores en la acción política de la polis. Habermas señalará al respecto que este juicio práctico, al ejercerse en el plano interactivo, exige la acción comunicativa o la interacción simbólica. En tal sentido, las normas consensuales y vinculantes son la base para el cumplimiento de las expectativas recíprocas. El acto educativo supone esta interactividad y reciprocidad de la que habla Habermas; no obstante, habría que incorporar otro elemento: el fin de este modo de vinculación en la praxis educativa no es el interés práctico sino el emancipatorio.

Este tipo de interés se basa en lo que Freire (1987) Ilama la concienciación y la capacidad de situarnos como sujetos históricos. Se trata de ejercer una verdadera praxis capaz de otorgar los medios necesarios para que los individuos se eleven a la categoría de sujetos históricos. Esto significa que el maestro no puede continuar ejerciendo su praxis como un técnico o administrador de currículo. Su labor debe estar encaminada a la emancipación, lo que exige la implementación de prácticas pedagógicas capaces de distinguir de manera inteligible las intencionalidades, las estrategias, los medios, los contextos y, especialmente, los aspectos constitutivos de los sujetos de la formación, en nuestro caso, los jóvenes, hombres y mujeres.

De este modo, asumir un tipo de currículo, incorporar la conveniencia de la integración y de la interdisciplinariedad, así como disponer de los medios y recursos necesarios para ejercer la práctica, se pueden convertir en un conjunto de racionalidades instrumentales, al decir de Habermas (1975), que quedan atrapadas en el vacío de la cotidianidad educativa si no se comprende que el horizonte de la praxis es la emancipación, la transformación, la producción de nuevos capitales culturales (Bourdieu, 2005), pensar de otro modo la sociedad y la cultura, fomentar la creación y la acción colectiva. En suma, se trata de un proceso de deconstrucción curricular en el que hay que poner en discusión preguntas como: ¿qué enseñar?, ¿para qué enseñar?, ¿para qué tipo de sujetos y sociedad?, ¿qué necesitan saber y hacer los sujetos introducidos en la universidad?, ¿cómo orientar lo que se quiere enseñar y lo que los sujetos quieren y necesitan aprender?, ¿qué niveles de vinculación se pueden favorecer para avanzar en el interés emancipatorio?

\section{Interdisciplina y subjetividad en el currículo praxeológico}

Se asume que el currículo actual, en el caso de las Instituciones de Educación Superior - IES - necesita reestructurarse, renovarse y deconstruirse para alcanzar 
el interés emancipatorio aludido, cuyo objeto sería el fenómeno, la comprensión de la realidad, la praxis (Ortega-Esteban, 2005). El currículo universitario - en la mayoría de casos - se sigue entendiendo desde su acepción convencional, esto es, la organización por contenidos disciplinares, niveles ascendentes, demarcación entre lo femenino y lo masculino, prácticas de poder basadas en el disciplinamiento del cuerpo y la mente, así como el ideal de la obediencia reinante en los imaginarios de la formación, entre otros atributos ampliamente estudiados por la pedagogía crítica, la filosofía y la sociología, que se distancian radicalmente de las intenciones de un currículo praxeológico que busca la formación integral del ser humano.

Desde distintos ángulos, autores como Torres (1991) y López-Jiménez (2011), han propuesto que la organización curricular tiene muchas posibilidades de ejercerse, entre ellas, los núcleos problémicos, los proyectos de aula, los proyectos de áreas y todo un repertorio de alternativas que ante todo fomenten la experiencia, es decir, que afecten la vida de los que participan en el acto educativo. Asimismo, BarragánGiraldo (2007) manifiesta que las universidades tienen la tarea fundamental de comprender y darle nuevos significados a las prácticas que caracterizan sus contextos y que estos "parecen estar mediados por la relación entre el desarrollo humano sustentable, la tecnología, el conocimiento y el desarrollo económico" (Barragán, 2007, p. 211).

En esta línea es relevante rescatar la concepción sobre currículo que expone Stenhouse: "El currículo es un intento de comunicar los principios esenciales de una propuesta educativa de tal forma que quede abierta al escrutinio crítico y pueda ser traducida efectivamente en práctica" $(1991$, p. 6). Por tal razón, decir que el currículo es un intento es establecer que el currículo nunca será acabado y definitivo y que debe ser considerado como una apuesta, un, precisamente, intento de educación a través de la comunicación, transmisión y legitimización de las consideraciones educativas que se intentan perpetuar desde los parámetros filosóficos de bueno, bello y verdadero del fenómeno humano a abordar desde la propuesta educativa, lo que lo deja abierto al debate y a los posibles cuestionamientos que se configuren en una acción concreta.

Al respecto, la interdisciplinariedad se convierte en un criterio y, a la vez, en un procedimiento concreto que puede favorecer esta deconstrucción curricular. La interdisciplinariedad como categoría remite a debates profundos en el orden de lo epistemológico, lo teórico y lo metodológico. Por ahora, baste señalar que el interés de la interdisciplinariedad en una perspectiva del currículo praxeológico es ampliar los niveles de comprensión de los fenómenos de los mundos natural, físico y social. Se trata de introducir conceptos, categorías, explicaciones -que transitan por disciplinas, campos de conocimiento y saberes del mundo ancestral y cotidianoque permiten pensar de otro modo el mundo, los sujetos, los saberes y los contextos para la formulación de alternativas creativas de cambio. 
Por último, el interés emancipatorio y la deconstrucción del currículo como posibilidad de reinvención de la escuela tiene una exigencia de gran trascendencia: el conocimiento profundo de los sujetos de la formación. Bajo las condiciones del cambio de época (Castells, 2004; Barbero, 2003), es claro que las expresiones de las subjetividades, comprendidas como formas de ser y estar en el mundo (Foucault, 2005), están pasando por una serie de mutaciones irrefrenables y hasta incomprensibles para las generaciones adultas. Asuntos como el cuerpo, la sexualidad, la comunicación, el consumo y las estéticas de la vida adquieren un lugar central en la vida de los jóvenes de hoy. Así, es evidente el tránsito de una subjetividad centrada en la disciplina escolarizada, los buenos modales y la obediencia, a una subjetividad que asume la experiencia, las trayectorias individuales y colectivas, el riesgo, la anticipación al mundo adulto y lo semiótico como bases para la construcción de sus propios mundos de vida.

A pesar de lo polémico que puede parecer este panorama y la preocupación que hoy se esgrime en el seno de la familia, la academia y la sociedad en torno a procesos como la juvenilización de la infancia y la juvenilización de los adultos, las relaciones sexuales tempranas, la violencia escolar, la incorporación de jóvenes en el conflicto social y armado, entre otros fenómenos, se puede concluir que la comprensión de estas mutaciones es de vital importancia para considerar los procesos de reestructuración curricular y los medios para lograrlo, teniendo como horizonte de sentido el interés emancipatorio. En consecuencia, las teorías del desarrollo evolutivo, las etapas de desarrollo, la estructuración psicobiológica de la mente y el cuerpo de los sujetos, claramente asumidas por el currículo convencional, requieren ser repensadas. Asuntos como el desarrollo en su concepción acumulativa y evolutiva deben ser reformulados atendiendo a conceptos emergentes de los estudios sociales y de la sociología de la juventud tales como: mundos de vida, trayectorias vitales, subjetividades políticas, estéticas, culturales, narrativas, hipernarrativas y biorresistencias, entre otros.

Lo anterior conlleva a entender que la búsqueda de sí mismo es una tarea ardua que hace que el individuo pueda devenir sujeto (Freire, 1980); sin embargo, es necesario que antes el individuo logre reconstruir su propia experiencia mediante un doble apartamiento que le posibilite asumir su libertad y resistirse a la total masificación consumista y enajenante que le ofrece la sociedad. En primera instancia, el sujeto debe apartarse del control comunitario que lo ancla en unas normatividades, unas representaciones, unas relaciones de poder y, en fin, una organización social que absorbe la experiencia vivida y le impide cualquier posibilidad de asumirse como sujeto personal. Este apartamiento conlleva un costo emocional y social altísimo: desgarramiento, estigmatización, descalificación, autoexclusión y sentimiento de pérdida de identidad. Ya no se trata de unirse $-\mathrm{y}$ de paso legitimarse socialmente- $\mathrm{a}$ una gran causa o bandera social o política, de lo que se trata ahora es de reivindicar el derecho a la existencia individual. Este deseo de individuación es lo que le posibilita 
subjetivarse, convertirse en actor social. En segundo lugar, el sujeto debe apartarse de las coacciones y moldeamientos de la sociedad de mercado que lo empujan a una vida para el consumo y la identificación con la masa, a la homogeneización y la desubjetivación.

De este modo, el currículo tiene que incluir en su propia lógica estos tránsitos y transiciones, los cuales no sólo implican considerar la naturaleza del mundo globalizado o la importancia de desarrollar competencias en los estudiantes para que se pongan al servicio del capitalismo transnacional global, sino articular las transformaciones ontológicas de estos sujetos para favorecer los procesos de cambio que requiere una sociedad con rezagos coloniales profundos de sexismo, patriarcalismo y racismo. Al respecto, la propuesta del currículo praxeológico se convierte en una oportunidad para avanzar en estos tres grandes propósitos en el interés de reinventar el currículo y fomentar prácticas creativas para la emancipación enmarcadas en la pedagogía praxeológica (Juliao, 2014).

Así las cosas, la temática consiste en centrar el análisis curricular en la categoría práctica pedagógica. Para dicho abordaje se deben tomar como ejes de comprensión el concepto de currículo, las competencias docentes, la interdisciplina, la práctica y saber pedagógico y la ontología. En términos generales, es asumir que la praxis curricular y el trabajo del profesor suponen una reflexión permanente "como responsable de la constitución del sentido de la vida de los individuos a él confiados" (Barragán-Giraldo, 2007, p. 215).

\section{Acerca de la pedagogía y las prácticas y el saber pedagógico}

Desde sus inicios, la pedagogía ha sido objeto de indagación por diversos campos de conocimiento, entre estos, la psicología y la filosofía, y ha centrado su interés en la elucidación conceptual de términos fundamentales para el discurso pedagógico tales como: formación, maestro, enseñanza, aprendizaje, didáctica, currículo, entre otros. Si bien estos aportes han dotado de sentido y significado a la pedagogía, es importante señalar la emergencia de dotar de su propio sentido a la pedagogía, es decir, de crear un lugar de discusión sin mediaciones de otras ciencias. Para ello, se propone recuperar las voces de los actores del proceso educativo, especialmente la de los maestros, como sujeto activo de los fenómenos escolares. En consecuencia, se trata desde los saberes de los docentes de dotar de significado y sentido a la práctica o el quehacer docente; reflexión que configurará lo que se denomina el saber del maestro, el cual, a su vez, nutrirá el campo de conocimiento denominado pedagogía.

Cuando el maestro logra evidenciar su quehacer cotidiano mediante el análisis de los componentes expuestos en el anterior apartado acerca de las prácticas pedagógicas, comienza un proceso de decodificación de las mismas, el cual le 
permite reflexionar sobre su ejercicio, resignificando sus saberes y formas de enseñanza. Así, el maestro, al realizar una práctica informada o autorreflexiva, logra dar cuenta del rol que cumplen sus prácticas pedagógicas en la configuración de nuevos sentidos/saberes escolares de manera que pueda desarrollar procesos de intervención motivados desde la relectura de su trabajo. Esto es lo que se Ilama el saber pedagógico.

Tal como se ha venido desarrollando, las prácticas pedagógicas se constituyen en el horizonte articular de las actividades académicas al contener y distribuir los saberes del maestro acerca de sus procesos de enseñanza-aprendizaje. En este sentido, el enfoque crítico entiende el currículo como proceso y producto aglutinador de dichas prácticas y saberes que sintetiza las posiciones epistemológicas, psicológicas y educativas que se pretenden desarrollar, lo cual define la práctica concreta de la enseñanza.

Esta situación conlleva a realizar una investigación de corte documental basada en la búsqueda, selección, registro, análisis e interpretación de documentos existentes en el área de la pedagogía con el fin de describir los fundamentos teórico-epistemológicos del currículo praxeológico y, de esta forma, determinar las características distintivas que proporcionen las herramientas necesarias que permitan diseñar y evaluar este tipo de currículo. Por tal razón, surge la emergencia de articular el currículo praxeológico con los modelos educativos de formación integral. Para esto se encuentra que los ejes de análisis son las posibles triangulaciones entre los constructos teóricos de Shirley Grundy, Jürgen Habermas, las concepciones y narrativas docentes sobre praxeología y, por último, las concepciones teóricas de un currículo praxeológico, con el fin de entender y comprender la pedagogía praxeológica (Juliao, 2014) como un referente teórico y práxico de nuevas formas de llevar un currículo a la práctica.

\section{Relación paradigmática curricular}

Para Grundy y Habermas, conocimiento y acción serán una misma cosa, por lo que el conocimiento se hace tal desde la autoformación. Y si asimismo se afirma que hay una vinculación directa entre acción y conocimiento, no se podría decir que los determinados intereses inferiores — prácticos y técnicos- se caracterizan por una heteronomía, sino que también serán constitutivos de conocimiento pues es innegable la acción en dichos intereses y su utilidad como condiciones de posibilidad de la objetividad y la veracidad de los enunciados que contienen. (Habermas, 1975). De esa manera, las teorías sobre currículo praxeológico, conocimiento y acción parten de una interpretación utópica de un hombre nuevo y de una sociedad diferente, aborda conceptos de persona, educación y sociedad claramente asumidos y diferenciados. El conocimiento hace parte de la concienciación del individuo que comprende que la cotidianeidad constituye saber y que la educación y la cultura son procesos de acción y trasformación continua. 
Lo sostenido anteriormente Ileva a contemplar y analizar saberes, imaginarios y narrativas docentes sobre su propia práctica en los que conocimiento y acción no son la misma cosa, son elementos constitutivos pero separados; en los que no se evidencia una vinculación directa de los intereses en la praxis, se encuentran separados los conceptos de subjetividad, interdisciplina y currículo de la acción de trasformación social.

\section{Análisis del currículo}

\section{El currículo desde el interés técnico}

Según Habermas, el interés técnico produce un saber instrumental en forma de explicaciones científicas aparentemente desinteresadas, cuya aplicación es el control de la naturaleza y las ventajas materiales de la producción. Se trata, en definitiva, de las ciencias empírico-analíticas. La teoría consta de conexiones hipotéticodeductivas de proposiciones que permiten deducir hipótesis legales pregnantes de contenido empírico (Habermas, 1975). El saber empírico-analítico es, por tanto, posible saber pronóstico.

De otro lado, el currículo técnico según Grundy se fundamenta en el interés técnico, esencialmente desde un interés por el control y la manipulación del ambiente. Este enfoque de diseño curricular supone que el educador producirá un educando que se comportará de acuerdo con la imagen esperada; para lograrlo deberá controlar tanto el ambiente de aprendizaje como el aprendiz (Grundy, 2004). Cuando prima un interés técnico, el estudiante carece de poder para poder determinar los objetivos de su aprendizaje; no obstante, puede ejercer un poder reactivo si se muestra no dispuesto o incapaz para participar en un ambiente de aprendizaje.

Esto conlleva a que, para un Ilamado currículo praxeológico, la fundamentación esencial y la razón de ser de sí mismo es pensar el concepto de ser humano y de mundo que se imprime en todas las prácticas educativas de la institución, es decir, que el aprendizaje debe estar orientado en sentido crítico de análisis y trasformación de la realidad (UNIMINUTO, 2014).

Sin embargo, se encuentra que el currículo para los docentes ${ }^{6}$, desde sus saberes, prácticas e imaginarios, es un documento que contiene una estructura de las directrices, fundamentos o bases filosóficas y teóricas que otorgan a una Institución de Educación Superior —IES - la intencionalidad de su apuesta formativa. Para

6 Este apartado está basado en los análisis de resultados de 11 grupos focales desarrollados con docentes universitarios en torno a las categorías iniciales del proyecto: saber pedagógico, práctica pedagógica, currículo, y su interacción e integración con los fundamentos de un currículo praxeológico. Los análisis surgen de un proceso categorial desarrollado a través del software ATLAS.ti. 
los docentes prima el interés técnico y la consistencia teórica en la construcción del currículo; se considera lo interdisciplinar sólo como un proceso para aclarar conceptos y se evidencia un completo desconocimiento de las dinámicas de subjetividad presentes en el mismo.

\section{El currículo desde el interés práctico}

Para Habermas, el interés práctico implica comprensión del significado de acciones concretas, de acciones realizadas en contextos culturales como la misma escuela. Esta comprensión, si bien implica otorgarles sentido a las acciones, también exige procesos de interacción. En las ciencias histórico-hermenéuticas es la comprensión de sentido lo que, en lugar de la observación, abre acceso a los hechos. El que comprende mantiene una comunicación entre los dos mundos, capta el contenido objetivo de lo transmitido por la tradición y al tiempo aplica la tradición a sí mismo y a su situación (Habermas, 1975).

Por otra parte, Grundy entiende el currículo desde el interés práctico cuando se resalta en él —el currículo-la acción o la práctica y no algún tipo de producto. La preocupación fundamental del profesor en este tipo de currículos es el aprendizaje, no la enseñanza; el aprendizaje supone no la producción de ciertos artefactos, sino la construcción de significado (Grundy, 2004). Por esto los profesores no sólo deberán preocuparse por comprender los contenidos de los objetivos prescritos, sino que deberán rechazar como contenidos educativos legítimos todo lo que no tenga como núcleo principal la construcción de significado del estudiante. Cuando la práctica está guiada por el interés práctico, la tarea será considerada como la interacción entre el lector y el autor para generar un significado en un acto de construcción que se toma completamente en serio.

Esto tiene como consecuencia que para un currículo praxeológico el reconocimiento de la diversidad de interacciones que acontecen entre los actores de su comunidad, con el conocimiento mismo y sus límites, con sus contextos y con el horizonte de sentido enunciado en la filosofía institucional, se convierten en proceso fundamental para el desarrollo del currículo desde un interés práctico (Uniminuto, 2014). Sin embargo, al realizar un análisis desde los saberes, prácticas, narrativas e imaginarios docentes ${ }^{7}$, la concepción de entender el currículo como Plan de estudios es permanente y es latente en los maestros. Estos consideran que el currículo es una apuesta del docente por brindar a los estudiantes conocimientos "importantes" para el desarrollo de su profesión. Los currículos entonces son ejercicios de práctica profesoral en los que el docente debe evidenciar lo que espera

7 La población de docentes pertenecientes a los grupos focales fue de 46 en relación con un total de 203 docentes que hacen parte de la Institución de Educación Superior que participó de la investigación "Competencias docentes, investigación y pedagogía praxeológica: ¿cómo se integran desde la vocación ontológica de ser sujeto?". 
realizar en su quehacer pedagógico.

\section{El currículo como interés emancipatorio}

Habermas identifica un tercer interés que busca la liberación, la emancipación, y conduce a las disciplinas critico-sociales. Lo llama interés emancipatorio. El interés emancipatorio pretende descubrir todas aquellas ataduras de la realidad, todas aquellas esclavitudes de las que todo ser humano es víctima más o menos inconsciente y de las que busca la mejor manera de romper con su yugo (Habermas, 1975). El interés emancipatorio posee un estatus derivado, es decir, garantiza la conexión del saber teórico con la práctica de la vida como un ámbito objetual que sólo surge bajo las condiciones de una comunicación sistemáticamente distorsionada y de una representación aparentemente legitimada (Habermas, 1975).

De esta forma, el currículo como praxis para Grundy (2004) busca comprometerse con el cambio de estructuras en las cuales se produce el aprendizaje, así como promover la crítica para afianzar la transformación. El interés emancipatorio tiene una dimensión ética y política: la dimensión ética significa una apuesta por un currículo en el cual los individuos, además de presentar las razones de sus acciones, establecen vínculos de cooperación y solidaridad; la dimensión política implica reconocimiento de la búsqueda de equidad y de igualdad (2004).

Por tal razón, un currículo praxeológico está basado en el conocimiento acumulado sobre los procesos educativos que acontecen en su comunidad académica para responder con una estructura capaz de atender pertinentemente a los desafíos, retos y necesidades de los contextos locales, regionales, nacionales e internacionales (UNIMINUTO, 2014). Adquiere la intencionalidad del currículo práxico de Grundy aquel que busca hacer emerger principalmente aquellas prácticas emancipadoras-transformadoras en las que las relaciones e interrelaciones se modifican sustancialmente cuando los miembros de la comunidad educativa tienen en cuenta tres principios básicos de una práctica pedagógica emancipadora: (i) los estudiantes deben ser participantes activos en el programa de aprendizaje; (ii) la experiencia de aprendizaje debe resultar significativa para el estudiante; y, (iii) la experiencia de aprendizaje debe ser correspondiente a su vocación ontológica y su proyecto de vida.

Sin embargo, este tipo de concepción desde los saberes, prácticas, narrativas e imaginarios de los docentes universitarios ${ }^{8}$ no evidencia un currículo como praxis; aunque se enuncia conocimiento sobre pedagogía praxeológica, los relatos

8 De la muestra seleccionada para conformar los grupos focales de docentes, el $41 \%$ son profesionales no licenciados que no poseían ningún curso, diplomado, posgrado o profundización en temas pedagógicos; el 28\% corresponde a profesionales no licenciados con formación en pedagogía, didáctica y educación; y el $31 \%$ corresponde a profesionales de las ciencias de la educación. 
y narrativas sobre su accionar en el aula de clase no muestran una aprehensión de los procesos significativos en los que se pueda asegurar la promoción de un pensamiento crítico y reflexivo que permita la trasformación social del entorno.

\section{El currículo praxeológico como comprensión critica de la realidad}

Un currículo praxeológico está orientado hacia la praxis. Su meta principal es desarrollar en el estudiante una comprensión crítica de la realidad social y el compromiso con la trasformación de las comunidades de una forma creativa, Poïesis (Juliao, 2015). Concibe la educación como un vehículo para lograr la concienciación y habilitación crítica del sujeto, transformar las maneras de ser y actuar con el contexto y, en este proceso, obtener como resultados la autoafirmación, autonomía y responsabilidad del sujeto (Juliao, 2014).

Un currículo praxeológico plantea que éste le debe permitir al estudiante alcanzar una conciencia crítica a través de su proceso educativo, lo que le permitirá no sólo comprender, entender e interpretar su realidad a través de la reflexión constante de sus prácticas, sino que logrará trascender en un proceso de transformación de su propia realidad. Es por eso que el modelo curricular praxeológico se orienta al desarrollo de la integralidad del sujeto con la intención de alcanzar su transformación y la de su entorno.

Para hacer realidad un currículo praxeológico se debe generar compromiso con el proceso de enseñanza-aprendizaje y estar dispuesto a participar activamente en la formaciónde estudiantes críticos y reflexivos. Al respecto, Juliao (2007) alega que un currículo praxeológico pretende un desarrollo pleno y multidimensional de las personas por medio de la integración coherente de los saberes — teoría- con sus acciones - praxis - en completa relación con los problemas sociales que deberá asumir el futuro profesional, además de los problemas que tienen que ver con el mejoramiento de la calidad de vida, la apuesta por una vida plena y virtuosa, y superar, de ese modo, los aspectos relacionados con la injusticia social, la opresión, la inconsciencia, la falta de ética.

El currículo praxeológico se centra en el paradigma de la educación y la pedagogía social, la cual está relativamente vinculada a la evolución de los problemas reales que afectan a la sociedad y en particular a la institución universitaria. Hoy en día se espera que desde el currículo praxeológico el paradigma se centre en la propia interpretación que el docente haga de su contexto en cuanto a los problemas que afectan a su universidad e impactan su práctica docente. Sin embargo, sea cual fuera el paradigma a desarrollar, el currículo praxeológico como alternativa de interpretación de la realidad y la educación, siguiendo a Pineda (2016), debe propender por:

* La práctica pedagógica debe constituir el nodo preferente de cambio curricular. 
* Es preciso encauzar la aplicación de ecosistemas para el aprendizaje para que los conocimientos se reconstruyan y se apropien de los cambios e impacten a la sociedad, una ecología del aprendizaje.

* Establecer espacios y procesos para el trabajo cooperativo en comunidades de aprendizaje y de práctica en docentes y estudiantes.

* El proceso educativo debe ajustarse y adaptarse a las dimensiones de la realidad, al contexto y a las necesidades del currículo como praxis social, un currículo praxeológico.

El currículo praxeológico establece cómo el sujeto puede estructurar pensamientos — logos — a medida que está inmerso en la acción — praxis—y, de tal modo, poder constituir sus acciones y lograr deducir postulados a priori, argumentos y acciones que posibiliten las decisiones del docente en la reflexión sobre su práctica. La estructura mental que se crea es una conducta consciente que se moviliza y se rige por la voluntad trasformada en accionar docente, es la reacción consciente y subjetiva que emerge del querer alcanzar el final de los objetivos propuestos. La estructura mental que se va construyendo está mediada por los estímulos y circunstancias del ambiente de aprendizaje; por tal razón, la experiencia praxeológica es única e irrepetible, cada encuentro entre los actores del proceso de enseñanza-aprendizaje es particular y responde a los intereses de cada sujeto que está influenciado por sus formas de ser y de interpretar la realidad.

La praxeología se presenta como un discurso — logos - sobre una práctica particular, intencionada y significante — praxis_- construido después de una seria reflexión como un procedimiento de objetivación científica de la práctica o de la acción, como una teoría de ésta. Por el tipo de análisis que se realiza, pretende que dicha práctica sea más consciente de su lenguaje, de su funcionamiento y de lo que en ella está en juego, sobre todo del proceso social en el cual el practicante está implicado y del proyecto de intervención que construye para modificar dicho proceso. Todo esto con el fin de acrecentar su pertinencia y su eficacia liberadora. (Juliao, 2002, p. 95)

Así, para el currículo praxeológico, el abordaje de las subjetividades es un acto esencial del proceso educativo ya que es a través de ellas —las subjetividades-que el sujeto se apropia de la realidad. Para el currículo praxeológico las percepciones, los argumentos y los lenguajes propios de los sujetos son las herramientas esenciales que configuran sus puntos de vista, las intencionalidades, propósito y cauce de la clase, el currículo, la práctica y la interpretación colectiva de la realidad (Pineda, 2016). Estas subjetividades de los implicados en el proceso de enseñanza-aprendizaje son las que cargan de valor la experiencia del saber, conocer e interpretar la realidad.

[L]a pedagogía será algo serio cuando la actividad del maestro se defina desde la actividad del estudiante, y la enseñanza a partir del aprendizaje. Solo en ese caso podemos hablar de praxeología pedagógica, porque solo si lo hacemos así podemos generar un campo propicio a los aprendizajes, a modalidades de aprendizaje que no se limiten ni terminen en producciones — clases, tutorías, ambientes de aprendizaje, tareas, exámenes, etc.- sino que se planteen como fines inmanentes que expresan la actividad autónoma del sujeto en su comprensión de sí mismo, de 
los otros y del mundo. Y esto, simplemente, porque la acción y la palabra —praxis y lexis-son manifestación/revelación del sujeto, no de algunas de sus características secundarias, sino de su cualidad esencial, de su mismo ser. (Juliao, 2011. p. 159)

Atender a las subjetividades desde un currículo praxeológico es encargarse de posibilidades de disponer nuevos modos de efectuar el acto educativo (Pineda, 2016). El currículo praxeológico propende por el establecimiento de ecosistemas para el aprendizaje que fomenten trasformaciones personales y sociales, genera conocimiento y saberes para una vida consciente a través de la reflexión, la crítica, la significación y la pertinencia. El acto educativo entonces incita a un proceso reflexivo y crítico sobre su propia práctica —la del docente y la de los estudiantes- que los conduzca a una real utilidad flexible de los saberes por medio de los componentes del enfoque praxeológico: ver, juzgar, actuar y devolución creativa (Juliao, 2011). Estos componentes responden a un análisis crítico, una interpretación, una reelaboración de la realidad y una evaluación y prospectiva que ejerce el sujeto en su camino de trasformación. El proceso adopta una metodología autobiográfica que busca impregnar de propias narrativas el acto educativo, Juliao (2011) lo manifiesta de la siguiente forma:

Se puede asumir que todo proceso formativo es primero un acto existencial, cuya finalidad es provocar y actuar la propia vida. En este sentido, se requiere que el quehacer praxeológico comprenda una perspectiva autobiográfica, tanto para revelar rupturas y encuentros en la existencia personal del sujeto, como para percibir y asumir la aparición creativa de un proyecto personal de vida y/o (sic) profesión. (p. 160)

El currículo praxeológico posibilita la realización por parte de los actores educativos de ecuaciones intertemporales que permiten establecer una imagen de sí mismos en el presente inmediato y su futuro próximo. Tales ecuaciones intertemporales es posible hacerlas porque el enfoque praxeológico posibilita tomar decisiones a partir de dialogar constantemente entre las necesidades presentes y las necesidades futuras; el currículo praxeológico permite la disposición de ciudadanos críticos que construyen tejido social responsable. El currículo praxeológico establece otros modos de educación mediante una visión cultural y social, a través de la inclusión social, respetando sus formas de interpretación y configuración de la realidad y fomentando la necesidad de una trasformación social por medio de la reflexión del propio actuar.

Las premisas asociadas a las características de la pedagogía praxeológica contribuyen al cambio de las creencias y permiten condiciones favorables a su implementación. El hecho de que los maestros reconocen cada vez más la importancia de responsabilizar al aprendiz de su propio aprendizaje, la implementación cada vez mayor de enfoques colaborativos, el creciente lugar que asume el rol regulador de la evaluación formativa e incluso la evolución de las nuevas tecnologías de la información y la comunicación son condiciones que permitirán a los maestros 
considerar con mayor confianza la aplicación de los principios de la pedagogía del dominio y la gestión del aprendizaje. (Juliao, 2014. p. 290)

Son muchos los retos que debe afrontar un currículo praxeológico. Uno de ellos radica en la disposición ontológica de sus ejecutantes quienes llevan la responsabilidad de la deconstrucción y desaprendizaje de esquemas individualistas, poco creativos y sin prospectiva social que han sido constantemente difundidos por las prácticas pedagógicas tradicionales (Pineda, 2015).

\section{Conclusión}

Asumir el currículo praxeológico como otra forma de hacer educación desde la discusión y pertinencia del currículo, el carácter interdisciplinario de la educación y la posibilidad de vincular a los sujetos — subjetividades - en el proceso educativo, es una apuesta disruptiva que adopta y asume una formación integral.

Un modelo educativo praxeológico, centrado en la formación integral, entendida como la educación que pretende el desarrollo armónico de todas las dimensiones de la persona. Este modelo se ordena, entonces, a la conformación de una persona que integra el saber —-teoría— con el actuar — praxis—. (Juliao, 2007. p. 215)

De esta manera se genera una apuesta permanente por la lectura de la realidad a través de un paradigma cualitativo que se preocupa por la comprensión de las realidades y contextos sociales por medio de una hermenéutica de conjunto que considera la subjetividad, el currículo como constructo social y la interdisciplinariedad como factores de intervención en la construcción del proyecto de vida y de dimensiones humanas por parte de los actores educativos (Juliao, 2015). En esta conceptualización es que se ubica el enfoque praxeológico (Juliao, 2011) que busca ofrecer esa visión de conjunto de la realidad mediante la construcción discursiva de la práctica de sus actores — para Juliao (2015) en esto consiste el actuar consciente-. No obstante, es importante aclarar que la pedagogía praxeológica no es un acercamiento sin rigurosidad a la realidad sino que, al contrario, implica lecturas de procesos sociales, individuales y culturales que afectan el currículo -intencionalidad educativa—, lo trasforman y lo mutan constantemente:

La clave de lectura praxis-teoría no es una aproximación sin más a la realidad. Si bien el primer interés del proceso es el perfeccionamiento de una práctica que poco a poco se hace más consciente, ello no significa la ausencia de dispositivos o conocimientos previos [...] [S]i bien se requiere de un conjunto de elementos previos a la teoría, la plataforma teórica final es el resultado de esa reflexión crítica sobre la práctica desarrollada en un primer momento. (Juliao, 2015. p. 138)

La vinculación de las reflexiones sobre currículo, práctica pedagógica y saber pedagógico instauran una manera diferente de hacer educación, es considerar al estudiante como sujeto de su propio aprendizaje; es decir, en la generación de la 
vocación ontológica de ser sujeto se construye una experiencia educativa enmarcada en la posibilidad del sujeto de dejar huella histórica, de mejorar su calidad de vida y de propender por una sociedad con compromiso social. El currículo, la interdisciplina y la subjetividad son esenciales para la generación de proyectos de vida trascendentes y conscientes de su responsabilidad con la realidad.

No hay educación liberadora si no se siente la necesidad de liberarse de algo o alguien; no hay educación transformadora si no se experimenta un deseo y una posibilidad de cambio personal y social. No se requiere pensar del mismo modo ni estar de acuerdo con un modelo ideal, ni acogerse a las normas pretendidamente científicas, ni siquiera poseer una alternativa ya diseñada; basta compartir una utopía para sortear las limitaciones del aquí y el ahora, y creer que la educación puede responsabilizarse de convertir dicha utopía en realidad. Desde esta perspectiva praxeológica, la educación no puede ser considerada al margen de la sociedad en la que interviene. La pedagogía praxeológica es así, también, pedagogía social y puede conducirnos a otra educación. (Juliao, 2015, p. 16)

El momento coyuntural político y social que vive nuestro continente permite la posibilidad de repensar el proceso educativo, el giro actual en sus prácticas sociales y culturales es una expresión de resistencia y es un llamado a pensarnos heurísticamente desde nuestra tierra, desde nuestros sujetos y desde nuestra propia cultura. Es allí donde un currículo praxeológico como práctica social, centrada en el sujeto, la cultura, la sociedad y la educación como eje transversal, es posible; un currículo praxeológico, un currículo pensado desde el trópico.

Recibido: 9 de septiembre 2015

Aceptado: 27 de noviembre 2015

Cómo citar: Martínez, E. (2016). El currículo praxeológico como interés de conocimiento emancipatorio. Praxis Pedagógica, n. ${ }^{\circ} 18,11-25$ 